Randomised controlled trial

\section{Initial choice of medication has little effect on short-term or long-term outcome for most patients with Parkinson's disease}

10.1136/ebmed-2014-110115

\section{Robert A Hauser}

Department of Neurology, University of South Florida, Tampa, Florida, USA

Correspondence to: Dr Robert A Hauser, Department of Neurology, University of South Florida, 4001 E. Fletcher Ave. 6th floor, Tampa, FL 33613, USA; rhauser@health.usf.edu

Commentary on: Gray R, Ives N, Rick C, et al.; PD Med Collaborative Group. Long-term effectiveness of dopamine agonists and monoamine oxidase B inhibitors compared with levodopa as initial treatment for Parkinson's disease (PD MED): a large, openlabel, pragmatic randomised trial. Lancet 2014:384:1196-205

\section{Context}

Levodopa is generally considered the most effective medication to improve motor features of Parkinson's disease (PD). However, long-term therapy is associated with the development of motor fluctuations and dyskinesias. Alternatives to levodopa include monoamine oxidase type B inhibitors (MAOBI) and dopamine agonists (eg, ropinirole, pramipexole and rotigotine). Clinicians may choose to initiate PD therapy with these 'levodopa sparing' agents in an effort to reduce the development of levodopa-associated treatment complications, especially in younger patients who are at highest risk.

\section{Methods}

The PD MED trial was a large, open label study in which newly diagnosed patients with PD were randomised to initial treatment with levodopa, a dopamine agonist or an MAOBI, then followed for up to 7 years. Either levodopa or MAOBI could be eliminated from the randomisation options at the discretion of the investigator. The other medications could be added if adequate control was not achieved with the assigned medication or if adverse events occurred. The primary outcome measure was the mobility subscale of the 39-item PD Questionnaire (PDQ-39). Patients completed study forms by post at baseline, 6 months and annually. Disease status was assessed at baseline and annual clinic visits. The study was designed to detect a six-point difference across groups in the PDQ-39 mobility score at any one timepoint, which was considered the minimal clinically important difference.

\section{Findings}

In total, 1620 patients were randomised from November 2000 to December 2009 with a median follow-up of 3 years. A high percentage of patients randomised to each drug class eventually received drugs from other classes. Over the 7-year follow-up, higher percentages of patients randomised to MAOBI (72\%) or dopamine agonists (50\%) stopped allocated treatment than patients randomised to levodopa (7\%). Results indicated that PDQ-39 mobility scores were not significantly different comparing levodopa and levodopa sparing medications at any one time point. However, the average score over the observation period was 1.8 points better with levodopa than with levodopa sparing medications ( $p=0.005)$. On the other hand, levodopa was associated with a greater risk of dyskinesia $(\mathrm{HR}=1.52$ and $\mathrm{p}=0.03)$. Additional results indicated that PDQ-39 mobility scores averaged 1.4 points better for MAOBI than dopamine agonists ( $\mathrm{p}=0.05)$, with similar risk for dyskinesia. Notably, the differences in PDQ-39 mobility scores between levodopa and levodopa sparing medications, as well as between MAOBI inhibitors and dopamine agonists, were substantially smaller than the predefined minimal clinically important difference of six points.

\section{Commentary}

This was an open label study and results could potentially be affected by preconceived notions and biases. Another limitation is that it was not truly randomised because investigators could exclude a treatment arm (levodopa or MAOBI) if they felt it was inappropriate for the patient. Patients who were excluded from initial treatment with levodopa were 9 years younger than patients who were not (62 years, $n=214$ vs 71 years and $n=1406)$. Further, the overall study population appears to have been older than would be considered typical for the PD population.

The results of the PD MED trial are similar to what has been observed in randomised double-blind trials comparing levodopa and dopamine agonists for initial therapy for PD. In one 48-month double-blind study (CALM-PD), patients were randomised to initial treatment with pramipexole (a dopamine agonist) or levodopa, with additional open label levodopa added as needed. ${ }^{1}$ The primary analysis found that the risk of dyskinesia was significantly less in the pramipexole group. However, disabling dyskinesia was uncommon and not different between groups. Additionally, Parkinson's scores (Unified Parkinson Disease Rating Scale) showed greater improvement in the levodopa group. Quality of life scores were not significantly different between the two groups.

The greatest uncertainty regarding initial choice of medication in early PD is how best to treat young individuals. Unfortunately, PD MED shines little light on this question as only $12 \%$ of patients were younger than 60 years. Nonetheless, even an analysis of these patients might be worthwhile.

\section{Implications for practice}

It seems likely that the PD MED study will have little effect on current treatment of PD. Clinicians who favour levodopa sparing strategies will continue to do so and those who rely almost exclusively on levodopa will continue to do so. For most patients, the initial medication choice appears to have little effect on long-term outcome. In part this may be because most patients are treated with multiple medications over time and differences in treatment may diminish. In addition, if side effects are encountered with one medication, it can be replaced with another.

Uncertainty still exists as to whether levodopa sparing strategies might be helpful for younger patients ( $<60$ years). These patients are at high risk of dyskinesia, a sensitivity to dopaminergic medications that persists indefinitely. Whether this problem can be meaningfully ameliorated in this subpopulation through the use of levodopa sparing strategies and whether this would have a significant positive impact on long-term quality of life, is as yet unknown.

Competing interests $\mathrm{RAH}$ has received honoraria or consulting fees from Teva Pharmaceuticals, Boehringer Ingelheim, and UCB.

Provenance and peer review Commissioned; internally peer reviewed.

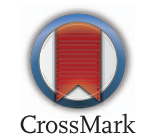

\section{Reference}

1. Holloway RG, Shoulson I, Fahn S, et al. Pramipexole vs levodopa as initial treatment for Parkinson disease: a 4-year randomized controlled trial. Arch Neurol 2004;61:1044-53. 